\title{
Vascular disease prevalence in diabetic patients in China: standardised comparison with the 14 centres in the WHO multinational study of vascular disease in diabetes
}

\author{
Z.S. Chi' ${ }^{1}$, E.T. Lee ${ }^{2}$, M.Lu ${ }^{2}$ H. Keen ${ }^{3}$, P.H. Bennett ${ }^{4}$ and the WHO Multinational Study Group* \\ ${ }^{1}$ Department of Endocrinology, Capital Hospital, Beijing, People's Republic of China \\ ${ }^{2}$ Centre for American Indian Health Research and Department of Biostatistics and Epidemiology, College of Public Health, \\ University of Oklahoma Health Sciences Centre, Oklahoma City, USA \\ ${ }^{3}$ Unit for Metabolic Medicine, Guy's Hospital, London, UK \\ ${ }^{4}$ Phoenix Epidemiology and Clinical Research Branch, NIDDK, Phoenix, Arizona, USA
}

\section{Abstract}

Aims/hypothesis. Rates of vascular complications of diabetes in a cohort of mainland Chinese patients with diabetes, ascertained and examined by similar methodology, are compared with those of the WHO Multinational Study of Vascular Disease in Diabetes (WHO MSVDD).

Methods. The standardised procedures carried out in the WHO MSVDD were followed in assembling and examining a Chinese cohort of 447 diabetic patients recruited in Beijing and Tianjin [2].

Results. Compared with the WHO MSVDD centres, the Chinese cohort was slightly older, had a shorter duration of known diabetes and had fewer insulintreated patients. Arterial pressure, total blood cholesterol and body mass index were substantially lower. Large vessel disease rate for age, sex and duration adjusted data (17.9\%) was about half that of the com- bined WHO MSVDD centres $(33.5 \% p<0.001)$. However, retinopathy $(47.4 \%$ vs $35.8 \% p<0.001)$ and proteinuria $(57.1$ vs $24.9 \% p<0.001)$ rates were significantly higher.

Conclusion/interpretation. Relatively low arterial pressures and blood cholesterol are likely contributors to the notably low arterial disease rates in this Chinese diabetic cohort; they reflect low rates in the Chinese mainland general population and resemble the Tokyo and Hong Kong centres of the WHO MSVDD. The high rates of retinopathy and proteinuria could relate to later diagnosis, degree of hyperglycaemia and/or increased susceptibiltiy to microangiopathy. [Diabetologia (2001) 44 [Suppl 2]: S 82-S 86]

Keywords Macrovascular disease, microvascular disease, diabetes, China, multinational study, arterial pressure, plasma cholesterol, risk factors.
We compare the prevalence of vascular disease and associated risk factors in a Chinese diabetic cohort with the results from the WHO Multinational Study of Vascular Disease in Diabetes (WHO MSVDD) which involved 14 centres from 13 countries [3].

Corresponding author: Elisa T. Lee, PhD, College of Public Health, University of Oklahoma, Health Sciences CentreP.O. Box 26901, Oklahoma City, OK 73190

* See reference 3 for list of investigators

Abbreviations: WHO MSVDD, World Health Organization Multinational Study of Vascular Disease in Diabetes; LVD, large vessel disease.

\section{Methods}

During the early 1980's, 447 diabetic patients (202 men and 245 women) aged 35 to 54 years were recruited in Beijing and Tianjin [1] exactly following the protocol [2, 3] established for the WHO MSVDD prevalence survey in 14 centres worldwide. The Chinese patients were selected by a randomising procedure from outpatient clinics in six hospitals, into the prescribed age and diabetes duration categories. The questionnaire, standardised examination procedures and centralised laboratory methods used in the WHO MSVDD survey [2, 3] were followed closely. Forward stepwise linear logistic regression and multivariate analysis [4] was carried out to identify important associations for large vessel disease and small vessel disease of eye and kidney. A $p$ value of 0.05 or less was considered statistically significant. 
Table 1. Comparison of frequency (\%) of risk factors in Chinese and pooled WHO cohorts

\begin{tabular}{|c|c|c|c|c|}
\hline & \multicolumn{2}{|c|}{ Chinese cohort } & \multicolumn{2}{|c|}{ Pooled WHO cohorts ${ }^{\mathrm{a}}$} \\
\hline & $\begin{array}{l}\operatorname{men} \\
\%\end{array}$ & $\begin{array}{l}\text { women } \\
\%\end{array}$ & $\begin{array}{l}\text { men } \\
\%\end{array}$ & $\begin{array}{l}\text { women } \\
\%\end{array}$ \\
\hline \multicolumn{5}{|l|}{ Age (years) } \\
\hline$<42$ & 15.3 & 24.6 & 26.0 & 25.8 \\
\hline $42-48$ & 41.1 & 40.6 & 33.3 & 34.4 \\
\hline 49 & 43.6 & 34.8 & 40.7 & 39.8 \\
\hline \multicolumn{5}{|l|}{$\begin{array}{l}\text { Duration of diabetes } \\
\text { (years) }\end{array}$} \\
\hline$<7$ & 54.7 & 61.5 & 39.8 & 40.8 \\
\hline $7-13$ & 27.4 & 25.0 & 32.6 & 32.9 \\
\hline 14 & 17.9 & 13.5 & 27.5 & 26.3 \\
\hline \multicolumn{5}{|l|}{$\begin{array}{l}\text { Age at diagnosis } \\
\text { (years) }\end{array}$} \\
\hline$<25$ & 4.5 & 4.9 & 10.1 & 10.5 \\
\hline $25-34$ & 20.4 & 23.0 & 27.4 & 26.3 \\
\hline $35-44$ & 49.8 & 47.3 & 42.0 & 42.4 \\
\hline 45 & 25.4 & 24.7 & 20.5 & 20.7 \\
\hline \multicolumn{5}{|c|}{ Treatment for diabetes } \\
\hline Diet alone & 12.9 & 10.2 & 16.5 & 15.2 \\
\hline Oral drugs & 54.0 & 60.8 & 42.0 & 44.4 \\
\hline Insulin & 33.2 & 29.0 & 41.5 & 40.5 \\
\hline \multicolumn{5}{|c|}{ Plasma cholesterol (mmol/l) } \\
\hline$<4.65$ & 51.7 & 38.3 & 18.6 & 16.7 \\
\hline $4.65-6.69$ & 47.2 & 51.9 & 56.2 & 57.6 \\
\hline $6.70-7.72$ & 1.1 & 5.6 & 13.9 & 14.7 \\
\hline 7.73 & 0.0 & 4.2 & 7.9 & 8.1 \\
\hline \multicolumn{5}{|c|}{ Systolic blood pressure $(\mathrm{mmHg})$} \\
\hline$<120$ & 23.0 & 29.8 & 18.0 & 17.4 \\
\hline $120-139$ & 44.0 & 37.2 & 38.4 & 33.1 \\
\hline $140-159$ & 23.5 & 21.1 & 26.9 & 28.3 \\
\hline $160-179$ & 6.5 & 9.1 & 11.1 & 13.8 \\
\hline 180 & 3.0 & 2.9 & 5.6 & 7.2 \\
\hline Hypertension & 27.7 & 30.6 & 31.8 & 36.0 \\
\hline $\begin{array}{l}\text { on blood-pressure- } \\
\text { lowering drug }\end{array}$ & 20.4 & 22.7 & 15.4 & 20.8 \\
\hline Smoking & 39.2 & 13.6 & 43.3 & 18.8 \\
\hline Mean BMI $\left(\mathrm{kg} / \mathrm{m}^{2}\right)$ & 23.4 & 24.1 & 25.6 & 27.6 \\
\hline
\end{tabular}

${ }^{a}$ from WHO MSVDD [3]

\section{Results}

Table 1 compares the distribution of a number of clinical characteristics of the Chinese cohort with those of the pooled WHO MSVDD cohorts from the 14 centres. The Chinese cohort had a higher proportion of older males, of those older at diagnosis and of those with shorter duration of diabetes in both sexes. Fewer Chinese subjects had a systolic pressure greater than $140 \mathrm{mmHg}$ and their mean pressure was lower than that of the WHO cohort. The Chinese also had a lower prevalence of hypertension, and of all patients classified as having hypertension, $74 \%$ were taking blood pressure-lowering drugs compared to $53 \%$ in the WHO cohort. More plasma cholesterol concentrations were below $4.65 \mathrm{mmol}$ in the Chinese than in the WHO cohorts. The proportion using insulin or diet alone was lower, therefore the use of oral drugs
Table 2. Comparison of prevalence (\%) of large vessel disease in Chinese and pooled WHO cohorts

\begin{tabular}{|c|c|c|c|c|}
\hline & \multicolumn{2}{|c|}{ Chinese cohort } & \multicolumn{2}{|c|}{$\begin{array}{l}\text { Pooled WHO } \\
\text { cohorts }^{\mathrm{a}}\end{array}$} \\
\hline & $\begin{array}{l}\text { men } \\
\%(n)\end{array}$ & $\begin{array}{l}\text { women } \\
\%(n)\end{array}$ & $\begin{array}{l}\text { men } \\
\%\end{array}$ & $\begin{array}{l}\text { women } \\
\%\end{array}$ \\
\hline Angina pectoris & $3.5(7)$ & $4.1(10)$ & 7.4 & 12.4 \\
\hline $\begin{array}{l}\text { Possible myocardial } \\
\text { infarction }\end{array}$ & $3.5(7)$ & $2.0(5)$ & 8.3 & 8.3 \\
\hline ECG coronary possible & $5.4(11)$ & $10.2(25)$ & 13.1 & 18.4 \\
\hline ECG coronary probable & $0.5(1)$ & $0.8(2)$ & 4.7 & 4.4 \\
\hline Stroke & $4.0(8)$ & $2.9(7)$ & 2.3 & 2.6 \\
\hline Intermittent claudication & $1.0(2)$ & $1.2(3)$ & 1.6 & 1.4 \\
\hline $\begin{array}{l}\text { Lower-extremity } \\
\text { amputation/gangrene }\end{array}$ & $1.0(2)$ & $0.4(1)$ & 2.2 & 1.2 \\
\hline Total large vessel disease & $15.3(31)$ & $20.0(49)$ & 29.5 & 37.4 \\
\hline
\end{tabular}

${ }^{a}$ from WHO MSVDD [3]

was proportionately higher in the Chinese cohort. Only in Tokyo was the mean BMI lower than in the Chinese cohort.

China had much lower rates of angina pectoris, possible myocardial infarction, ECG coronary (possible or probable), lower-extremity amputation and/or gangrene and total large vessel disease (LVD) than the WHO cohorts. Ranked against the 14 individual centres, China had the lowest prevalence of LVD in women and the second-lowest in men. Only stroke prevalence was slightly higher in the Chinese sample than in the pooled WHO cohort. As in the other WHO centres, total LVD prevalence in women exceeded that in men (Table 2).

Prevalence of small vessel disease (SVD) eye was much higher in the Chinese than in the pooled cohorts in almost all sex or duration groups and ranked higher than all the individual centers when duration of diabetes was less than 14 years (Table 3). Adjusting for sex and duration, all grades of retinopathy affected $47.4 \%$ of the Chinese compared with $35.8 \%$ of the pooled cohorts $(p<0.001)$.

Rates of light and heavy clinical proteinuria (SVD kidney) were also much higher than in the pooled cohorts in all duration groups and for both sexes. China had the highest prevalence of proteinuria of all WHO centers for men in all duration groups and for women with the exception of Zagreb. Total proteinuria rates, adjusted for sex and duration, were $57.1 \%$ in the Chinese and $24.9 \%$ in the combined WHO cohorts $(p<0.001)$.

Stepwise linear logistic regression analysis (Table 4) identifies independent associations for LVD, SVD eye and kidney. All degrees of retinopathy were combined into an any retinopathy category and light and heavy proteinuria combined into a nephropathy category. Included in the regression model were age at examination, duration of diabetes, systolic 
Table 3. Comparison of prevalence (\%) of small vessel disease of eye and kidney in Chinese and pooled WHO cohorts by duration

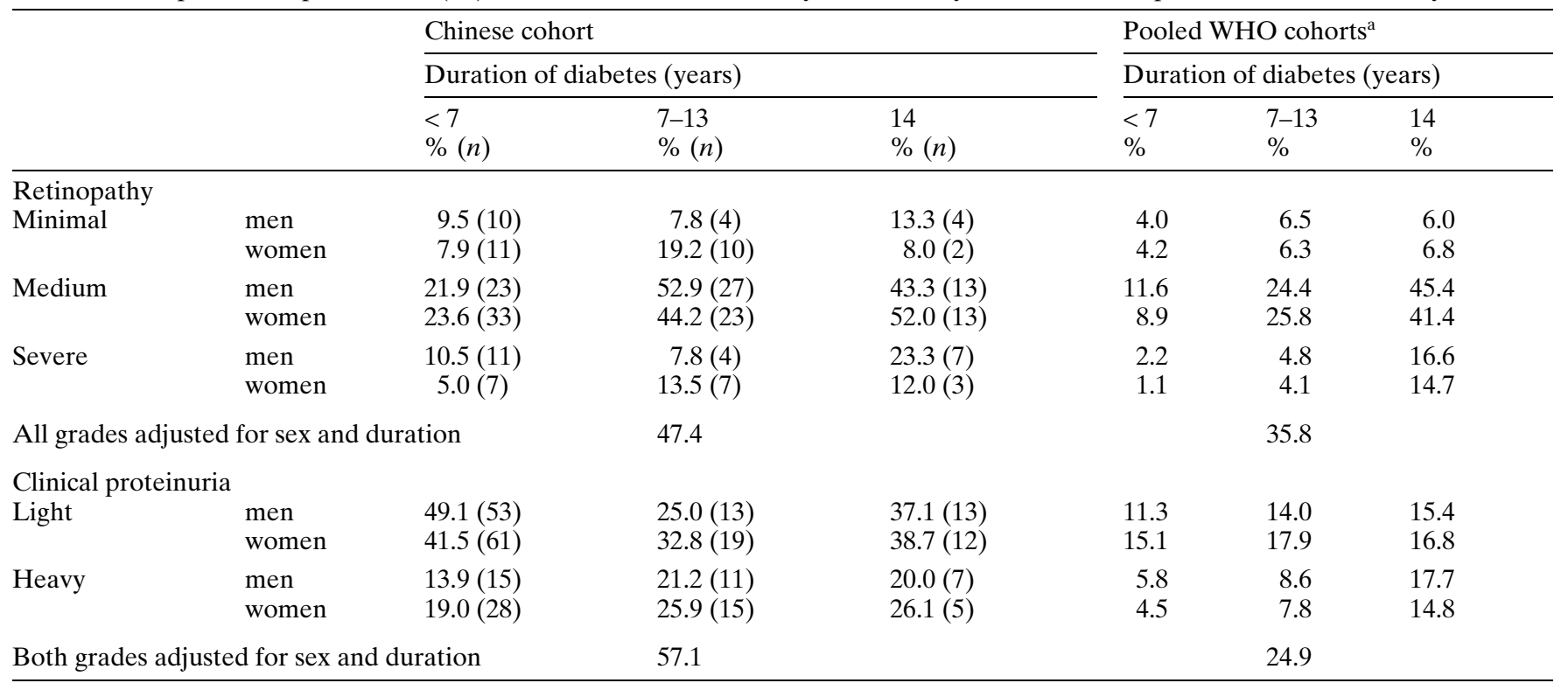

a from WHO MSVDD [3]

Table 4. Standardised coefficients of risk factors for large and small vessel disease from stepwise logistic regression in Chinese and WHO cohorts

\begin{tabular}{|c|c|c|c|c|}
\hline & \multicolumn{2}{|c|}{ Chinese cohort } & \multicolumn{2}{|c|}{$\begin{array}{l}\text { Pooled WHO } \\
\text { cohorts }^{\mathrm{c}}\end{array}$} \\
\hline & men & women & men & women \\
\hline \multicolumn{5}{|l|}{ Large vessel disease } \\
\hline Age (years) & 0.16 & 0.08 & $0.35^{\mathrm{a}}$ & $0.16^{\mathrm{a}}$ \\
\hline Duration of diabetes (years) & 0.09 & $0.24^{\mathrm{b}}$ & $0.16^{\mathrm{a}}$ & 0.08 \\
\hline Systolic blood pressure $(\mathrm{mmHg})$ & -0.08 & 0.12 & $0.29^{\mathrm{a}}$ & $0.34^{\mathrm{a}}$ \\
\hline BMI $\left(\mathrm{kg} / \mathrm{m}^{2}\right)$ & 0.21 & 0.15 & $0.17^{\mathrm{a}}$ & $0.22^{\mathrm{a}}$ \\
\hline Smoking & -0.01 & 0.10 & 0.06 & 0.02 \\
\hline Treatment for diabetes & 0.13 & 0.06 & 0.03 & 0.03 \\
\hline Plasma cholesterol (mg/dl) & 0.12 & $0.15^{\mathrm{a}}$ & $0.18^{\mathrm{a}}$ & 0.06 \\
\hline \multicolumn{5}{|l|}{ Any retinopathy } \\
\hline Age (years) & 0.11 & 0.04 & -0.03 & $0.04^{\mathrm{b}}$ \\
\hline Duration of diabetes (years) & $0.25^{\mathrm{a}}$ & $0.41^{\mathrm{a}}$ & $0.33^{\mathrm{a}}$ & $0.33^{\mathrm{a}}$ \\
\hline Systolic blood pressure $(\mathrm{mmHg})$ & 0.03 & $0.14+$ & $0.15^{\mathrm{a}}$ & $0.12^{\mathrm{a}}$ \\
\hline BMI $\left(\mathrm{kg} / \mathrm{m}^{2}\right)$ & -0.12 & -0.05 & $-0.08^{\mathrm{a}}$ & $0.04^{\mathrm{a}}$ \\
\hline Smoking & 0.15 & 0.08 & 0.02 & 0.02 \\
\hline Treatment for diabetes & $0.22^{\mathrm{b}}$ & 0.04 & $0.18^{\mathrm{a}}$ & $0.16^{\mathrm{a}}$ \\
\hline Plasma cholesterol (mg/dl) & -0.05 & 0.12 & 0.01 & 0.01 \\
\hline \multicolumn{5}{|l|}{ Clinical proteinuria (light or heavy) } \\
\hline Age (years) & 0.07 & 0.04 & -0.02 & -0.03 \\
\hline Duration of diabetes (years) & -0.07 & 0.02 & $0.13^{\mathrm{a}}$ & $0.09^{\mathrm{a}}$ \\
\hline Systolic blood pressure $(\mathrm{mmHg})$ & $0.34^{\mathrm{a}}$ & $0.30^{\mathrm{a}}$ & $0.22^{\mathrm{a}}$ & $0.17^{\mathrm{a}}$ \\
\hline BMI $\left(\mathrm{kg} / \mathrm{m}^{2}\right)$ & 0.14 & 0.01 & 0.01 & 0.03 \\
\hline Smoking & 0.08 & -0.01 & $0.05^{\mathrm{a}}$ & 0.00 \\
\hline Treatment fo & 0.02 & 0.07 & 0.03 & $0.04^{\mathrm{b}}$ \\
\hline Plasma cholesterol (mg/dl) & 0.05 & $-0.19^{\mathrm{b}}$ & $0.04^{\mathrm{b}}$ & $0.10^{\mathrm{a}}$ \\
\hline
\end{tabular}

Smoking: 0-no, 1-yes. Treatment for diabetes: 0-diet alone, 1 -oral drugs, 2 -insulin

${ }^{\mathrm{a}} p<0.01,{ }^{\mathrm{b}} p<0.05$,

${ }^{\mathrm{c}}$ from WHO MSVDD [3] blood pressure, BMI, plasma cholesterol, smoking status and treatment for diabetes.

For LVD, only diabetes duration and plasma cholesterol concentrations in women were statistically significant. Age, systolic pressure and BMI, significant in both sexes in the pooled WHO cohorts, failed to qualify in the Chinese cohort. When hypertension as a category was used instead of systolic pressure in the regression model, it emerged as significantly related in men (standardised coefficient $=0.22$, $p<0.05$ ), and suggestively related in women (standardised coefficient $=0.16, p<0.10$ ).

For retinopathy (SVD eye), known duration of diabetes emerged as significant in both sexes as in the pooled WHO sample. "Intensity" of treatment (graded insulin, oral agents, diet alone) was significantly related in men and systolic pressure approached statistical significance in women.

As in the pooled cohorts, systolic blood pressure in the Chinese cohort was strongly associated with proteinuria in both sexes but diabetes duration showed no association.

\section{Discussion}

The diabetic patients in the Chinese cohort differed from those in the pooled WHO cohorts [3] with a much lower prevalence of LVD, stroke being the only exception. Overall, prevalence of LVD was less than half that of the pooled cohorts and resembled the low rates in Hong Kong and Tokyo though differing sharply from the higher prevalences reported by the South Asian (Indian) cohort [3]. Low coronary heart disease prevalence has also been reported in 
other studies of diabetes in indigenous Chinese people $[5,6]$ which reflects the low rates in the general Chinese population.

This low LVD prevalence in the Chinese cohort could be related to the lower arterial pressures and perhaps indirectly to the proportion of patients using blood pressure-lowering drugs. Low rates of hypertension and LVD were also found in the Tokyo and Hong Kong cohorts [3]. The strikingly low values of total plasma cholesterol in the Chinese cohort is also likely to be of major importance. There were few subjects at the upper segment of the pooled cohort range for cholesterol and a high proportion in the lowest segment of the range. The Tokyo and Hong Kong centres in the WHO MSVDD showed the same phenomenon with both low cholesterol concentrations and LVD prevalence. Relatively low smoking rates and BMI could also be 'protective' cardiovascular factors.

In contrast to LVD, the reported prevalences of SVD of eye and kidney, as shown by ophthalmoscopic evidence of retinopathy and clinical tests for urinary protein, were higher in the Chinese cohort than in any of the 14 WHO centres, including those in Asia when known diabetes duration was less than 14 years. Estimates of retinopathy prevalence based on different examiners using direct ophthalmoscopy must be treated with caution. Despite the standardisation of the method of examination and the simple descriptive reporting of findings the contribution of an unrecognized locality bias to systematic difference between centres cannot be excluded. However, limited data from cross-sampling and other reports of similar high rates of retinopathy and proteinuria in Chinese patients with diabetes [5-9], support the validity of the high retinopathy rates in this Chinese cohort.

Duration of diabetes was found to be an important association of retinopathy and its association with the 'intensity' of antidiabetic treatment, an indication of the surrogate role of the latter for higher blood glucose concentrations and suggesting that poorer glycaemic control could have contributed to the high rates of microvascular disease. The link between systolic blood pressure and retinopathy was found to be suggestive in women only but arterial pressures were generally low. Given that the Chinese cohort had a relatively short known duration of diabetes, a low proportion of patients using insulin, and low average systolic blood pressure, other factors are more likely to explain the high prevalence of retinopathy.

Clinical proteinuria was also found with very high frequency in this Chinese diabetic sample. Almost two thirds of the cohort were reported as proteinuric (light and heavy) in the first 7 years after diagnosis. These high rates continue to rise with increasing time from diagnosis but more slowly than in the pooled cohorts [3]. This is consistent with longer periods of unrecognized diabetes before diagnosis in the
Chinese and supported by the high, apparently early retinopathy rates. In a considerable proportion of people with Type II (non-insulin-dependent) diabetes mellitus, proteinuria is related to renal disease other than, or in addition to, diabetic nephropathy [11]. A high prevalence of heavy proteinuria was also reported in the Tokyo WHO MSVDD cohort [3] but low rates in one other study in China [10]. Systolic blood pressure was associated with clinical proteinuria in the Chinese cohort but it is difficult to reconcile the low overall systolic pressure with the systematically higher prevalence of proteinuria. This could suggest other contributory pathogenic mechanisms or greater inherent renal susceptibility to diabetes in Chinese people.

In conclusion, in comparison with the 14 centres of the WHO MSVDD, diabetic patients in this Chinese cohort had a lower prevalence of LVD, resembling the Japanese and Hong Kong cohorts of the WHO MSVDD. This could be related to the low prevalence of hypertension and low average concentrations of plasma cholesterol in the Chinese cohort correlating with the low atherosclerosis risk of the general population of mainland China. In contrast, the Chinese cohort had a much higher prevalence of diabetic retinopathy and clinical proteinuria than the pooled WHO cohorts. Observer variation and patient selection could have contributed to these differences but the strikingly low rates of macrovascular disease and the high prevalence of retinopathy and proteinuria reported calls for further objective study.

\section{References}

1. Beijing and Tianjin Diabetic Research Cooperation Group (1981) Study of complications in diabetes mellitus according to the WHO protocol. Chin Med J (Chin) 20: 513

2. Jarrett RJ, Keen H and Grabauskas V (1979) The WHO Multinational Study of Vascular Disease in Diabetes: 1. General Description. Diabetes Care 2: 175-182

3. Diabetes Drafting Group (1985) Prevalence of small vessel and large vessel disease in diabetic patients from 14 centres. The World Health Organization Multinational Study of Vascular Disease in Diabetes. Diabetologia 28: 615-640

4. SAS Technical Report P-200, SAS/STAT Software: CALIS and LOGISTIC Procedures, Release 6.04, SAS Institute Inc., Cary, North Carolina

5. Chi ZS (1983) Some aspects of diabetes in the People's Republic of China: Part 1. A perspective from Beijing (Peking). In: Mann JI, Pyorala K and Teuscher A (Eds). Diabetes in Epidemiological Perspective. Curchill Livingstone, Edinburgh, London, Melbourne, New York, pp 87-96

6. Hu YH, Pan XR, Liu PA, Li GW, Howard BV, Bennett PH (1991) Coronary heart disease and diabetic retinopathy in newly diagnosed diabetes in Da Qing, China: the Dai Qing IGT and Diabetes Study. Acta Diabetologica 28: 169-173

7. Zhu XX, Zhong XL (1983) Some aspects of diabetes in the People's Republic of China: Part 2. A perspective from Shanghai. In: Mann JI, Pyorala K and Teuscher A (Eds). 
Diabetes in Epidemiological Perspective. Edinburgh, London, Melbourne, New York, pp 87-96

8. Wang SH (1937) Diabetes mellitus: an analysis of 347 cases; incidence, symptoms, examination, and complications. Chin Med J 51: 159

9. Chi ZS, Dai QL, Hu SX et al (1986) Study on the community control of diabetes mellitus and its complications in the People's Republic of China. International Diabetes Federation Bulletin 32: 103-106
10. Pan XR (1980) Complications of diabetes mellitus. Chinese Journal of Internal Medicine (Chin) 19: 83

11. Parving HH, Gall MA, Skott P, Jorgensen HE, Jorgensen F, Larsen S (1990) Prevalence and causes of albuminuria in non-insulin-dependent diabetic (NIDDM) patients. Kidney Int 37: 243

12. Chen Z, Peto R, Collins R, MacMahon S, Lu J, Li W (1991) Serum cholesterol concentration and coronary heart disease in population with low cholesterol concentrations. BMJ 303: 276-282 